\title{
Prolonged Slow Negative Wave in Primary Sensory Evoked Potentials of Dog Cerebral Cortex
}

\author{
By \\ Kozo Ohsaki and Kitsuya Iwama \\ From the Department of Physiology, University of \\ Kanazawa, Medical School, Kanazawa
}

(Received for publication, January 4, 1961)

Since Iwama and Jasper ${ }^{11}$ reported the action of $\gamma$-aminobutyric acid (GABA) upon the primary sensory evoked potential of the cerebral cortex, many works have been done on this subject. It is now generally known that only the 10-30 msec surface-negative wave of the evoked potential is diminished by topical application of GABA, leaving the initial positive wave intact or augmented. However, when a detailed observation is made on the evoked potential under the action of GABA in non-anesthetized animals, it is very often found that a prolonged surface-negative wave $100-150 \mathrm{msec}$ in duration appears following the initial surface-positive wave.

Since no extensive studies on the slow negative wave of the evoked potential under the influence of GABA have been available, it was attempted to disclose properties of this sort of the evoked activity. In the course of the study, however, it was found that a similar prolonged negative wave could be produced as a component of the sensory evoked potential without application of GABA in non-anesthetized animals.

While this study was being carried out, the paper by Pearlman and others ${ }^{21}$ dealing with the slow negative wav in the visually evoked cortical potentials called our attention. The slow negative wave studied by them seems to be essentially identical with that reported in this paper. Though the experimental findinge obtained in the present study are in good agreement with those of the previous workers, it seems still worth while publishing this paper, because many points which remained unattacked have been elucidated in this study.

\section{METHOD}

All experiments were performed on adult dogs. Ether anesthesia was used during early stages of the operations, and then the animals were kept immobilized. by $\mathrm{d}$-tubocurarine chloride $(0.2-0.3 \mathrm{mg} / \mathrm{kg} / \mathrm{hr}$. intravenously) and maintained on

大崎 晃三, 岩間 吉也 
artificial respiration. Evoled potentials of the cerebral cortex were usually obtained by stimulating the thalamic somatosensory relay nuclei (n. ventralis posterolateralis and $n$. ventralis posteromedialis) with a concentric bipolar electrode $0.5 \mathrm{~mm}$ in diameter, which was inserted stereotaxically consulting the atlas of the dog's brain. ${ }^{3)}$ Stimuli were rectangular pulses of brief duration less than 10 volts in strength. Recording electrodes were a silver wire with a ball tip of $1 \mathrm{~mm}$ diameter for the cortical surface and a glass pipette (10 $\mu$ in tip diameter) filled with physiological saline for the deeper layers of the cortex. The reference electrode was a screw fixed to the skull over the frontal sinus. The electrical activities were amplified with condenser-coupled amplifiers (time constant: $1.4 \mathrm{sec}$ ) and led to a dual beam oscilloscope. Ink-writing tracings were simultaneously obtained.

The chemical agents used for modifying the evoked cortical potentials were $1 \% \mathrm{GABA}, 1 \% \mathrm{KCl}$ and $0.1 \%$ strychnine in physiological saline, all of which were applied topically to the exposed cortical surface.

\section{RESULTS}

It is well known that the primary evoked potential to a single shock in the sensory nucleus of the thalamus or peripheral sensory nerves consists of an initial surface-positive wave ( $\mathrm{P}$-wave) followed immediately by a surface-negative one (N-wave). In curarized, non-anesthetized dogs this diphasic primary complex was followed by a prolonged surface-negative slow wave ( $\mathrm{SN}$-wave). Its duration was as long as $150 \mathrm{msec}$ or more (Fig. 1, A; bottom record) and the amplitude measured about $500 \mu \mathrm{V}$ in the best condition. From some points of the sensorimotor cortex only the SN-wave was observed without being preceded by marked P-and/or N-waves (Fig. 1, B). The most excitable points for the SN-wave did not necessarily coincide with those for the primary complex. This suggests that the development of the $\mathrm{SN}$-wave is not directly related to that of the primary complex. However, the cortical areas giving rise to the $\mathrm{SN}$-wave in response to thalamic sensory stimulation were confined almost exclusively to the somatic afferent area which has been defined classically.

I. Amplitude of the $\mathrm{SN}$-wave as functions of intensity and frequency of stimulation

As illustrated in Fig. 1, among the primary complex and the SN-wave, the latter appeared first when the stimulus intensity was gradually increased from the sub-threshold level. Throughout the present study it was unfailingly found that the threshold for the SN-wave was slightly lower than that for the primary complex. The stimulus voltage for the maximal SN-wave was also lower than for the maximal primary complex. These observations are in complete agreement 
with the finding by Pearlman et al. As the primary response attained its maximal amplitude, the $\mathrm{SN}$-wave was definitely diminished (Fig. 1, A). This may

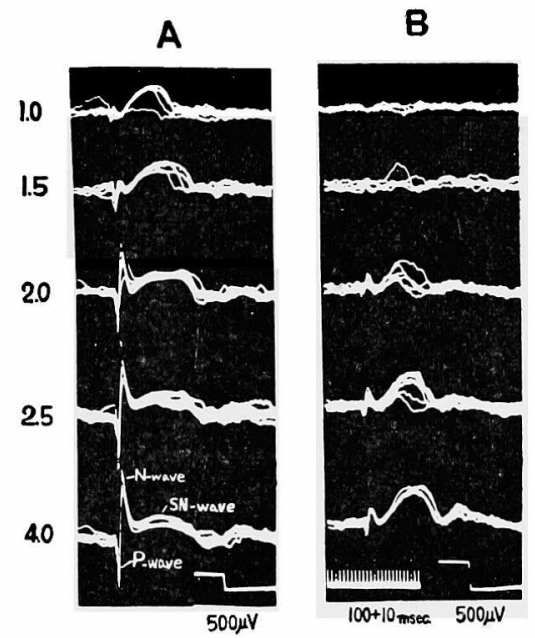

Fig. 1. Series of evoked cortical potentials to a single shock applied to thalamic VPL, showing effects of increasing stimulus intensity. Stimulus intensities are given to the right in arbitrary scale. All records were formed by superposition of 5 to 7 traces. A : Responses recorded from a most active site. Note, only SN-wave appeared to low voltage stimulation and as primary complex developed some masking effect on SN-wave obtained. B : Records obtained simultaneously with A from a locus a few mm apart from the point for A. Development of primary complex was less marked and its masking effect upon SN-wave was very little. Negativity upward in this and all subsequent figures.

be interpreted to mean that there is some masking effect of the primary complex upon the SN-wave, reducing the amplitude of the latter. This supposition is supported by the records in column B in Fig. 1 which were taken at a locus a few millimeters apart from the site most active for the primary complex. In this case the development of the primary complex is not marked, so that its masking effect upon the SN-wave was very little, resulting in a continuous increase of the $\mathrm{SN}$-wave with increasing the stimulus intensity.

When the thalamic sensory nucleus was repetitively stimulated, the amplitude of the SN-wave was notably affected. In Fig. 2 a representative instance is shown. In this experiment a series of stimuli with intensity just maximal for the SN-wave were applied to the thalamic VPL at varying frequencies. At a low frequency less than $0.5 / \mathrm{sec}$, the evoked SN-waves were considerably variable in amplitude (Fig. 2, A). By using a little higher frequency $(0.7-2 / \mathrm{sec})$, however, the variability of the $\mathrm{SN}$-wave was reduced and its amplitude was augmented (Fig. 2, B). When the stimulus frequency was further increased up to $3 / \mathrm{sec}$, 
the SN-wave responded in a waxing and waning manner (Fig. 2, C). The period of the waxing and waning of the amplitude was variable from case to case and

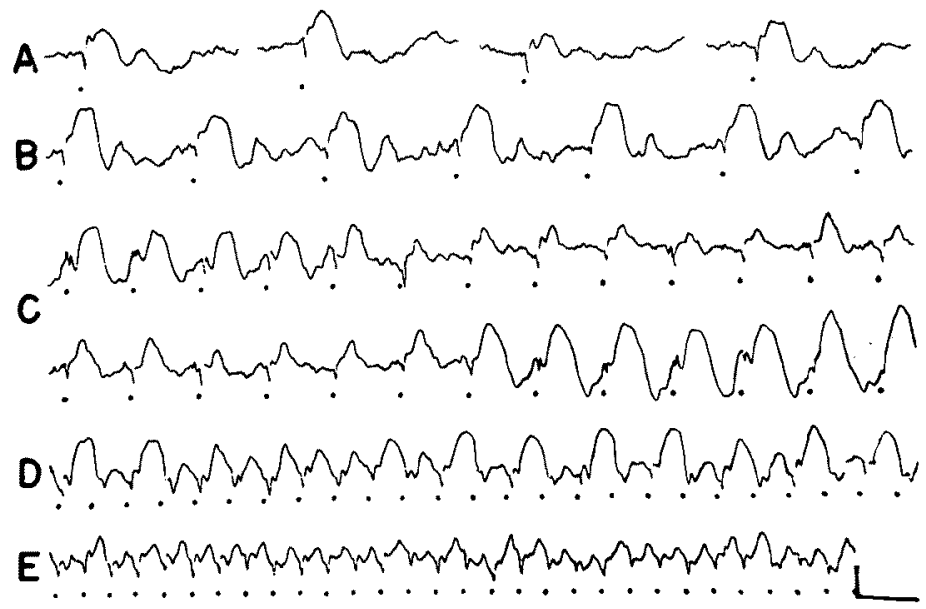

Fig. 2. Responses evoked by repetitive stimulation of VPL at $0.3 / \mathrm{sec}$ (A), 1.5 $/ \mathrm{sec}(\mathrm{B}), 3.7 / \mathrm{sec}(\mathrm{C}), 6.3 / \mathrm{sec}(\mathrm{D})$ and $8.5 / \mathrm{sec}(\mathrm{E})$. SN-waves were variable in $\mathrm{A}$ and were stabilized and slightly augmented in $\mathrm{B}$. In $\mathrm{C}, \mathrm{SN}$-waves appeared in a waxing and waning manner. Upper and lower records are continuous. In $\mathrm{D}$, high and low amplitude responses appeared alternately. With further high frequency (E) SNwaves were almost completely suppressed. Calibrations : $400 \mu \mathrm{V}$ and $250 \mathrm{msec}$.

also from trial to trial in one experiment. This response pattern to repetitive stimulation could be observed at a frequency as high as $7 /$ sec. In addition, it was observed with this stimulus frequency that the SN-wave appeared with high and low amplitude alternately at the waxing stage (Fig. 2, D). Using a further high frequency, the $\mathrm{SN}$-wave was almost completely suppressed, while the primary complex was still recognizable (Fig. 2, E).

II. Effects on the SN-wave of barbiturate anesthesia and of reticular formation stimulation

When the animals were left immobilized for several hours, it was a usual observation that EEG was altered from low voltage fast waves to high voltage slow waves. In association of such spontaneous change in EEG activity the amplitude of the SN-wave became very variable (Fig. 3, a), presumably because of the spontaneous slow waves prevailing in the background. Though such was the case with the $\mathrm{SN}$-waves, the primary complex remained comparatively stable or in some cases it was augmented. A similar observation could be made by administrating a small dose of barbiturate to curarized animals in full alertness. Usually an intravenous injection of 10 to $20 \mathrm{mg} / \mathrm{kg}$ barbiturate was sufficient to induce the spontaneous slow waves in EEG, making the evoked SN-wave unstable. 
A further addition of barbiturate up to $50 \mathrm{mg} / \mathrm{kg}$ in total dose was found to suppress the $\mathrm{SN}$-wave almost completely. Such a high dose of barbiturate suppressed the $\mathrm{N}$-wave considerably, and the $\mathrm{P}$-wave was also affected though to a lesser extent.

At the stage in which the SN-wave became unstable spontaneously or by injection of a small dose of barbiturate the effects of high frequency stimulation of the mesencephalic reticular formation (RF) upon the $\mathrm{SN}$-wave were examined. During stimulation of the RF the $\mathrm{P}$-wave was always suppressed and EEG altered
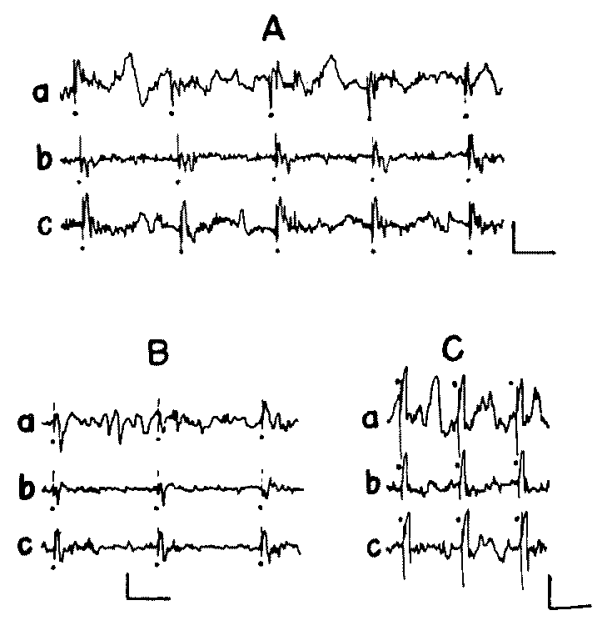

Fig. 3. Effects of RF-stimulation. Frequency of stimulation, 200/sec. Pulse width, $0.05-0.3 \mathrm{msec}$. Intensity, 5 volts. In each record, a : before, $\mathrm{b}$ : during and $\mathrm{c}$ : immediately after the stimulation. Sharp upward deflections indicated by dots are $\mathrm{N}$-waves. Calibrations : $400 \mu \mathrm{V}$ and 1 sec.

to show the arousal pattern. The effects on the $\mathrm{N}$ - and the $\mathrm{SN}$-waves, however, varied from one case to another. These effects could be classified into three types: 1) Both the $\mathrm{N}$ - and the SN-waves were suppressed (sample record not illustrated). Usually the effect on the latter was less marked than that on the former. 2) The SN-wave was suppressed with the $\mathrm{N}$-wave unaffected. In some cases the $\mathrm{SN}$-wave was gradually released from suppression during the stimulation (Fig. 3, A), and in others the suppression persisted as long as the stimulation continued (Fig. 3, B). 3) The $\mathrm{N}$-wave was remarkably suppressed, but the suppression of the SN-wave was very slight (Fig. 3, C). Taking these data altogether, it seems that the $\mathrm{SN}$-wave behaves under the reticular activating influence independently of the $\mathrm{N}$-wave.

When reticular stimulation was stopped, the potential waves, if suppressed during the stimulation, were recovered rapidly to the pre-stimulation level and were kept stabilized during the sustained EEG-arousal. In some cases a transient 
augmentation (rebound) of the evoked potential was observed in all the components immediately after the stimulation was withdrawn.

III. Modifications of the SN-wave by asphyxia and by topical application of $\mathrm{KCl}$, strychnine and GABA

Asphyxia: The susceptibility of the SN-wave to asphyxia was studied in comparison with that of the primary complex. With an animal immobilized with d-tubocurarine artificial respiration was stopped. Two to three minutes later the $\mathrm{N}$-wave was almost completely abolished, while the $\mathrm{P}$ - and the $\mathrm{SN}$ waves were still present though their amplitudes were reduced to about one half of the control and both were a little prolonged in duration (Fig. 4, B; upper trace). Three to four minutes after the commencement of asphyxia the SNwave disappeared (Fig. 4, B; lower trace). Then, artificial respiration was again started. Within one minute the $\mathrm{SN}$-wave recovered almost to the control level (Fig. 4, C). Five to seven minutes after resumption of ventilation, the entire potential complex returned completely to the control level (Fig. 4, D).
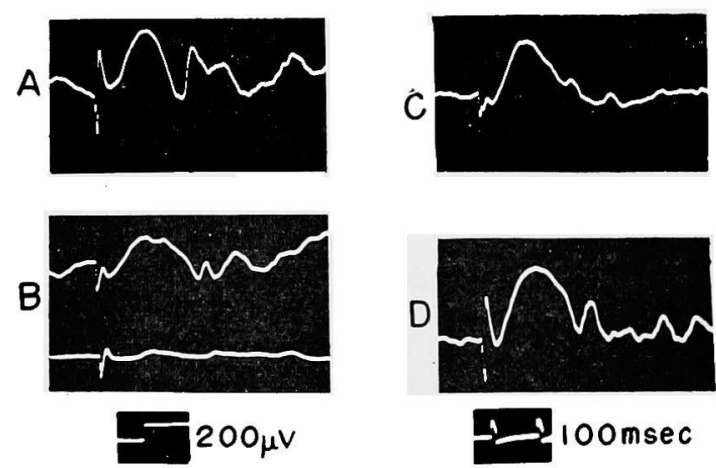

Fig. 4 Effects of asphyxia. A: Control response. B: Upper and lower traces are responses $3^{\prime}$ and $4^{\prime}$ after stopping artificial respiration, respectively. $\mathrm{C}: \mathrm{I}^{\prime}$ after resumption of respiration. SN-wave recovered more rapidly than primary complex. D : Full recovery of all components of evoked potential about $4^{\prime}$ after record C.

$\mathrm{KCl}$ : Suppression of the $\mathrm{SN}$-wave, similar to that seen in asphyxia, was obtained by topical application of $1 \% \mathrm{KCl}$ solution. Fig. 5, $\mathrm{B}_{1}$ is a record obtained two and a half minutes after $\mathrm{KCl}$ application. By this time the $\mathrm{N}$ wave had completely been abolished, while both the P-and the SN-waves remained almost unaffected. About five minutes later, the SN-wave was virtually abolished (Fig. 5, $\mathrm{B}_{2}$ ). After record. $\mathrm{B}_{2}$ was obtained, the cortical surface was rinsed with warm saline solution. Four minutes later, the $\mathrm{SN}$-wave recovered considerably though the primary complex was still suppressed (Fig. 5, $\mathrm{C}_{1}$ ). 
Within ten minutes the SN-wave completed a full recovery, but the primary complex remained reduced in size (Fig. 5, $\mathrm{C}_{2}$ ).
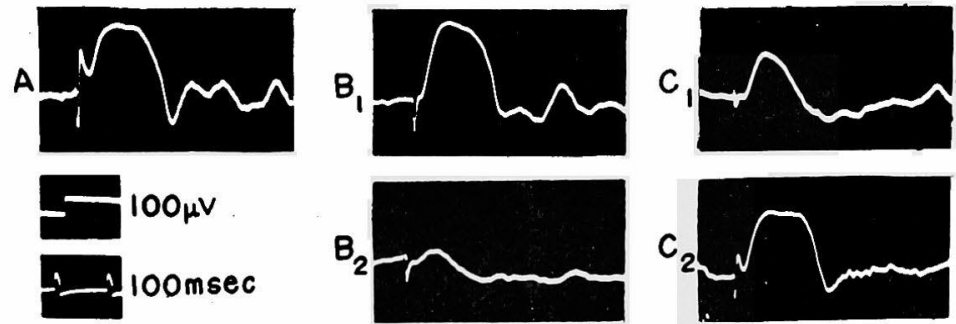

Fig. 5. Effects of topical $\mathrm{KCl} . \mathrm{A}$ : Control response. $\mathrm{B}_{1}$ and $\mathrm{B}_{3}: 2^{\prime} 30^{\prime \prime}$ and $5^{\prime} 45^{\prime \prime}$ after $\mathrm{KCl}$ treatment. N-wave was suppressed earlier than $\mathrm{SN}$-wave. $\mathrm{C}_{1}$ and $\mathrm{C}_{2}$ : Recovery at $3^{\prime} 42^{\prime \prime}$ and $10^{\prime}$, respectively, after washing off the brain. SN-wave recovered earlier than primary complex.

Strychnine: A solution of strychnine $0.1 \%$ in concentration was applied topically at a cortical point where the primary complex and the $\mathrm{SN}$-wave developed moderately (Fig. 6, A). With a lapse of time the primary complex, especially its $\mathrm{N}$-wave, was gradually augmented (Fig. 6, B), and finally it turned into a triphasic (positive-negative-positive) potential wave of very high amplitude (Fig. 6, C). The evoked potential so modified by local strychninization was very similar in wave form to the spontaneous strychnine spike which began to appear at the time of record $\mathrm{C}$ of Fig. 6 . In contrast to this, the $\mathrm{SN}$-wave could be traced on the declining phase of the augmented negative wave even at the final stage of strychninization. It seemed that the $\mathrm{SN}$-wave did not constitute a main component of the evoked potential augmented under the influence of strychnine.

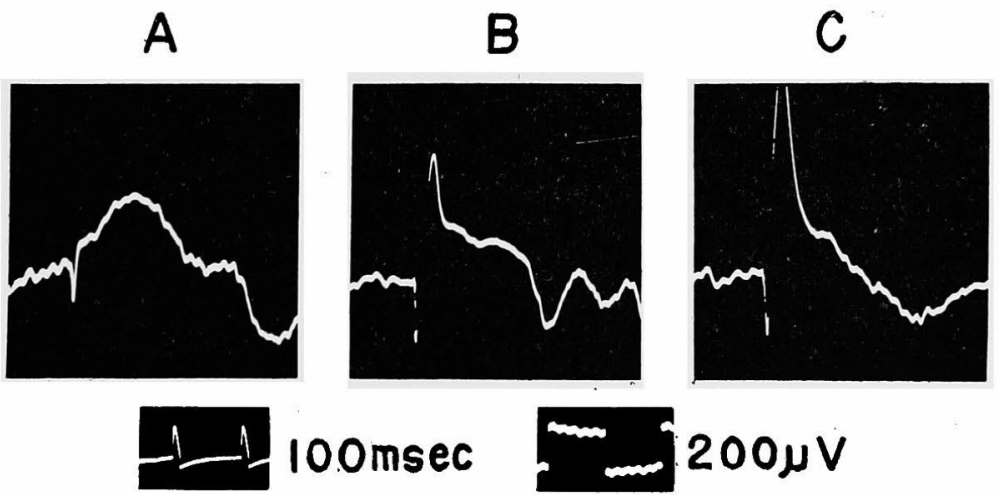

Fig. 6. Effects of topical strychnine. A : Control response. B and C : 5'30" and $11^{\prime}$ after local strychninization, respectively. At the time of record C, spontaneous strychnine spikes were observed. In C, SN-wave appeared as a notch on descending limb of augmented negative wave. 
GABA : The effects of topical application of GABA were examined in nonanesthetized animals showing the primary evoked potential with a good SNwave. An example of the results is shown in Fig. 7. In records A and B the upper and the lower traces show the responses from the surface and the deep layer $1 \mathrm{~mm}$ beneath the surface, respectively. The control response is shown in record $A$ and the response obtained 15 sec after topical application of 1\% GABA in record B. In conformity of the previous works, GABA promptly abolished the $\mathrm{N}$-wave and increased the $\mathrm{P}$-wave in amplitude and duration. In addition to this, it was observed that GABA augmented the SN-wave extremely. Corresponding to the modification of the surface response, the slow wave of positive polarity, which was found by the deep recording to be associated with the surface $\mathrm{SN}$-wave, was significantly increased in amplitude by GABA.
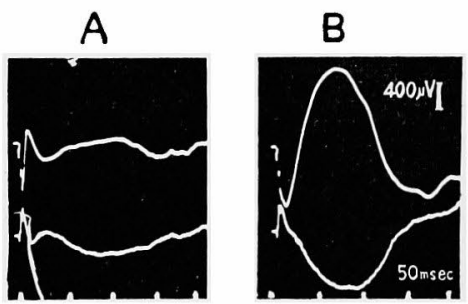

Fig. 7. Effects of topical GABA. In each set, upper and lower traces show surface response and response led from $1.0 \mathrm{~mm}$ beneath the surface, respectively. In $\mathrm{B}, \mathrm{SN}$-wave was extremely enhanced by surface application of GABA. Note simultaneous augmentation of deep slow positivity.

Augmentation of the SN-wave due to topical application of GABA, may be attributable partly to suppression of the $\mathrm{N}$-wave which, as stated in the previous section, masks a part of the SN-wave. If only the removal of the masking on the SN-wave is the cause of augmentation of the $\mathrm{SN}$-wave, it is expected that the SN-wave may also be enhanced, though transiently, when the $\mathrm{N}$-wave was progressively eliminated by $\mathrm{KCl}$ applied on the cortical surface. But such was not the case. On these bases it may be concluded that in addition to the removal of the masking, there must be an augmenting action of GABA upon the $\mathrm{SN}$-wave.

IV. Intracortical distribution of the $\mathrm{SN}$-wave

It has already been mentioned that the SN-wave is more resistant to the suppressing action of topically applied $\mathrm{KCl}$ than is the $\mathrm{N}$-wave of the primary complex. If the suppressing action manifests itself as $\mathrm{KCl}$ gradually diffuses from the surface to the deep layers, it is suggested that the $\mathrm{SN}$-wave originates from a deeper layer of the cortex than does the $\mathrm{N}$-wave. This is well supported by the intracortical recording of the evoked potential complex. 
Fig. 8 shows the responses recorded from various depths beneath the cortical surface. As the microelectrode was progressively inserted into the depth of the
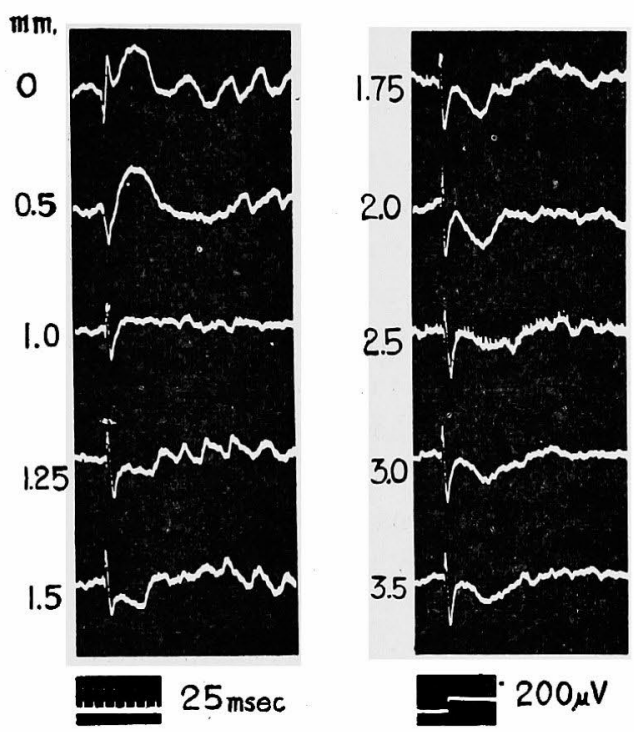

Fig. 8. Potential distribution in different depths of the cortex. Reversal point for SN-wave is located more deeply than for primary complex.

cortex, the first to be noted was the phase reversal of the primary complex. In this experiment the primary complex completed the reversal of phase before the depth of $1.0 \mathrm{~mm}$ was reached. This level was found to be the isoelectric point for the $\mathrm{SN}$-wave. At $1.25 \mathrm{~mm}$ beneath the surface the reversed. SN-wave, which is positive in sign, became evident. The depth at which the phase reversal of each component of the evoked potential occurred varied from case to case, but it was a consistent finding that the reversal point of the $\mathrm{SN}$-wave was located at a deeper layer than in the case of the primary complex. The deep slow wave of positive polarity attained the maximal amplitude about $2 \mathrm{~mm}$ below the surface, and then it decreased gradually when the microelectrode was thrust more deeply.

The fact that the phase reversal of the $\mathrm{SN}$-wave took place below the cortical surface suggests that it is an electrical potential localized within the cortex, and also that this potential is produced on the cortical neurones postsynaptically by thalamic afferent volleys.

\section{DISCUSSION}

In the present study it has been shown that the SN-wave is different from the primary complex in such points as threshold (Fig. 1), pharmacological 
selectivity (Figs. 5, 6, \& 7), intracortical potential distribution (Fig. 8) and so on. All these findings indicate clearly that the $\mathrm{SN}$-wave is a separate process from the primary complex and originates from some structures of a cortical layer deeper than that containing the element responsible for the $\mathrm{N}$-wave.

When an extensive study was made by Chang4) on the direct cortical response (DCR), he noticed that a slow negative wave followed a primary negative wave of short duration in response to a strong stimulus. According to Chang, the slow negative wave, called "the second component" of the DCR, had a higher threshold than the primary negative wave and it did not reverse its polarity when recorded from a deep layer $1 \mathrm{~mm}$ below the cortical surface. In contrary to Chang, Goldring and his associates ${ }^{5-7)}$ reported the slow negative wave of the DCR to reverse its polarity in the deeper layers of the cortex. A similar slow negative wave was also found by these workers as a component of the recruiting response of the cerebral cortex to repetitive stimulation of the thalamic intralaminar system. According to these workers, GABA, applied to the cortical surface, augments the slow negative wave in the DCR as well as in the recruiting response.

The characteristics of the slow negative wave as described by Goldring et al. well coincide with those of the SN-wave described in this paper, a suggestion being made that both are generated by the same mechanism. However, there seems some discrepancy between the slow wave of the DCR and the SN-wave of the primary evoked potential in regard to the intensity of stimulus required to elicit them. It has been mentioned in the previous section that the threshold of thalamic stimulation is lower for the SN-wave than for the primary positivenegative complex, while it has been reported by Chang and by Goldring et al. that in the DCR a stronger stimulus is required for the slow negative wave than for the primary negative wave. This discrepancy may be explained that direct stimulation of the cortical surface is a more favorable method of activation for the primary negative wave than for the slow negative wave, because the latter is considered to originate from the layer slightly deeper than those generating the former.

Discussion on the mechanism of potential generation of the SN-wave is rather a difficult problem. We have shown that the $\mathrm{SN}$-wave from the surface and the nearby superficial layers is associated with the slow wave of positive polarity in the deeper layers. This fact may indicate that there is an electric dipole which has the sink in the superficial layers against the source of the deeper layers. However, a question still remains to be answered which one, the superficial negativity or the deep positivity, is the locally produced active process. If the deep positivity represents an active process, the superficial negativity may be a passive phenomenon which is caused by spread of the current from the source in the deeper layers. If one follows this notion, the experimental finding 
that surface-applied GABA augments the superficial negativity and the deep positivity simultaneously must be explained as follows. Though GABA is placed on the cortical surface, it penetrates into the deeper layers and enhances the electrical activity of positive polarity arising therefrom, and in proportion to the enhancement of the deep positivity, the negativity of the surface and superficial layers will also be increased. However, this cannot be accepted in view of the finding of Yamamoto et $a l .^{8)}$ obtained with the cat cerebral cortex. They showed that if surface-applied GABA was maintained in a low concentration, its action was limited to the superficial layers within $1 \mathrm{~mm}$ below the surface. This may also be true of the dog cerebral cortex studied in the present experiment. Therefore, the GABA-induced augmentation of the SN-wave must be ascribed to enhanced activity of the nervous elements lying in the superficial layers where the $\mathrm{SN}$-wave is recorded with the negative polarity. This means that the enlargement of the deep positivity under the action of GABA is an event caused passively by the augmentation of the superficial negativity. This consideration will lead us to an inference that in the normal intact cortex, the $\mathrm{SN}$-wave of negative polarity originates as locally produced activity in the superficial layers, causing an electrotonically spread potential of positive polarity in the deeper layers.

The above-stated consideration is obviously based upon the assumption that GABA has an excitatory action upon some types of the electrical activity of the cerebral cortex. This seems not in agreement with the well-accepted notion that the action of GABA is an inhibitory nature. In recent times, however, some authors have suggested that the action of topically applied GABA on the cerebral cortex cannot be explained only in terms of inhibition. ${ }^{9-11}$ Moreover, as noticed by Iwama and Jasper under certain experimental conditions, a powerful enhancement of the sensory after-discharges followed local treatment of the cortical surface with GABA. In the present study it was occasionally observed that the sensory after-discharges, probably identical with the fast after-discharges of the intact cortex in Bremer's classification, ${ }^{12}$ ) were enhanced extremely by local treatment with GABA and sometimes it appeared like the seizure wave. Thus, it is probable that GABA does not always exert an inhibitory action on the cerebral cortex, at least in the non-anesthetized state.

\section{SUMMARY}

The cerebral cortex of curarized, non-anesthetized dogs responded to a single stimulation of the thalamic somatosensory nucleus with an evoked potential consisting of a primary positive-negative diphasic complex ( $\mathrm{P}$ - and $\mathrm{N}$-waves) followed by a slow negative wave (SN-wave) of $100-150 \mathrm{msec}$ in duration. The properties of the $\mathrm{SN}$-wave were studied in comparison with those of the $\mathrm{P}$ - and the $\mathrm{N}$-waves.

1. The localization of the SN-wave was limited within the somatosensory 
cortex, and its threshold was slightly lower than that of the primary complex. When the thalamic stimuli were applied at an appropriate frequency, the SNwave responded in a waxing and waning fashion.

2. In light barbiturate anesthesia, the $\mathrm{SN}$-wave became unstable more readily than did the primary complex. By high frequency stimulation of the midbrain reticular formation the $\mathrm{P}$-wave was more or less suppressed, but the effects on the $\mathrm{N}$ - and the $\mathrm{SN}$-waves were quite variable.

3. The $\mathrm{SN}$-wave was more resistant to systemic asphyxia or topically applied $\mathrm{KCl}$ than was the $\mathrm{N}$-wave. Strychnine augmented the primary complex, especially its $\mathrm{N}$-wave, whereas the $\mathrm{SN}$-wave was little affected. By topical treatment with GABA the $\mathrm{N}$-wave was abolished and the $\mathrm{SN}$-wave was extremely enhanced.

4. The intracortical recording revealed that the $\mathrm{SN}$-wave originated from some deeper points of the cortex than those producing the primary complex.

We are grateful to Dr. Tomoaki Asano for his invaluable suggestions in regard to preparing the manuscript. We wish also express our gratitude to Mr. Chosaburo Yamamoto for his valuable criticism during this investigation. A part of the expense of this work was defrayed by a grant of the Ministry of Education.

\section{References}

1) Iwama, K. \& Jasper, H.H., J. Physiol., 1957, 138, 365.

2) Pearlman, A.L., Goldring, S. \& O'Leary, J.L., Proc. Soc. Exp. Biol. Med., 1960. 103, 600.

3) Lim, R.K.S., Liu, C.N. \& Moffitt, R.L., Stereotaxic Atlas of the Dog's Brain. Charles C. Thomas, Springfield, 1959.

4) Chang, H.-T., J. Neurophysiol., 1951, 14, 1.

5) Goldring, S., O'Leary, J.L. \& Huang, S.H., EEG Clin. Neurophysiol., 1958, 10, 663.

6) Goldring, S., O'Leary. J.L. \& Pearlman, A.L., Proc. Soc. Exp. Biol. Med., 1959, 100, 429.

7) O'Leary, J.L. \& Goldring, S., Epilepsia, IVth series, 1959/60, 1, 561.

8) Yamamoto, C., Yuyama, T. \& Iwama, K., Jap. J. Physiol., 1959, 9, 160.

9) Takahashi, H., Nagashima, A. \& Arai, B., ibid., 1960, $10,106$.

10) Mahnke, J.H. \& Ward, A.A., Exptl. Neurol., 1960, 2, 311.

11) Iwama, K., Yamamoto, C. \& Ohsaki, K., Tohoku J. Exp. Med., 1960, 72, 366.

12) Bremer, F., Physiol. Rev., 1958, 38. 357. 